\title{
Programas de continuidad de cuidados: éxitos, fracasos y retos futuros
}

\author{
Ana González Rodríguez. \\ Centro de Salud Mental de Chamartín, Madrid
}

\begin{abstract}
Resumen
Los programas de continuidad de cuidados surgen a finales de los años 70 en EEUU, en respuesta a los problemas detectados durante del proceso de desintitucionalización de los hospitales psiquiátricos. Desde entonces, se han extendido por todo el mundo, con variaciones y peculiaridades según las regiones, convirtiéndose en piedra angular de la atención a las personas con enfermedad mental grave y persistente. En este artículo se revisa el origen de estos programas, su desarrollo a lo largo de más de treinta años, la filosofía que los ha guiado en su devenir, así como los éxitos y fracasos en su desarrollo.
\end{abstract}

Palabras claves: tratamiento asertivo comunitario; continuidad de cuidados; atención comunitaria en salud mental.

\begin{abstract}
Case management programs: successes, failures and future challenges. The case management programs arise in the late 70's in the U.S., in response to problems identified during the process of deinstitutionalization of psychiatric hospitals. Since then, the case management and the assertive community treatment programs have spread throughout the world, with variations and particularities in different regions, becoming the cornerstone of the community care for people with severe and persistent mental illness. This article reviews the origins of these programs in U.S., their development for over thirty years around the world, the philosophy and objectives that have guided its evolution, as well as successes and failures in their development.
\end{abstract}

Keywords: assertive community treatment; case management; community-oriented mental health care.

$\mathrm{L}$

os programas de continuidad de cuidados surgen a finales de los años 1970 en EEUU como respuesta al laberinto y caos en el que se convirtió la asistencia a los enfermos mentales graves y persistentes, tras el cierre de camas en los hospitales psiquiátricos. Como consecuencia del proceso de desinstitucionalización, iniciado a mediados de los años 1950 en EEUU, en menos de veinte años el número de camas se redujo drásticamente (National Institute of Mental Health, 1977), pero no fue paralelo a la creación de recursos alternativos apropiados en la comunidad, lo que se tradujo en un cuidado ineficiente, incluso en un abandono de los pacientes más graves. El Programa de Soporte Comunitario (Community Support Program) se diseña en colaboración con profesionales y entidades ciudadanas: familias, usuarios, investigadores, abogados, con la función de favorecer la creación y coordinación de una red diversificada de alojamientos, hogares, centros de rehabilitación y trabajo protegido, que permita a las personas con trastornos mentales funcionar en un nivel óptimo en la sociedad (Turner \& Ten Hoor, 1978).

El reconocimiento de la necesidad de ayudar a las personas con trastornos mentales graves a acceder y coordinar diferentes servicios psiquiátricos da lugar a nuevos programas comunitarios, que van a denominarse "case management" (en España se tradujo como programas de seguimiento, de apoyo comunitario o de continuidad de cuidados) y a un nuevo profesional de la salud mental, el "case manager" (coordinador de cuidados). Inicialmente su función era evaluar las necesidades del paciente, el desarrollo de un plan de prestaciones individualizado, la coordinación, la información y la negociación de los servicios, el seguimiento continuo del paciente durante este proceso y la defensa de sus derechos en el sistema. Es decir, coordinar, derivar e integrar varios servicios en un programa lo mejor confeccionado posible de acuerdo a las necesidades de cada paciente.

\section{Filosofia de los programas de continuidad de cuida- dos}

Todos los modelos de apoyo comunitario tienen como objetivo asegurar la continuidad de cuidados, reducir la hospitalización, mejorar el funcionamiento social y la calidad de vida de los pacientes. Para lograrlo son básicas cinco funciones: la evaluación, la planificación personalizada, la coordinación, el seguimiento y la defensa de los derechos de los pacientes. Siguiendo los principios esenciales de la continuidad de cuidados 
según Leona Bachrach (Bachrach, 1993), se resumirían en nueve:

Clima administrativo que apruebe, apoye y legitime los servicios para una población claramente definida. Es evidente que no puede haber continuidad de cuidados cuando los miembros de la pretendida población diana se mantienen fuera de las puertas del sistema y otros son atendidos en su lugar.

Accesibilidad. Es imposible la continuidad de cuidados si los pacientes no pueden alcanzar los servicios. Deben eliminarse las barreras geográficas, económicas y psicológicas que limitan el acceso.

Servicios integrales (amplios y completos), pues no hay continuidad si no se dispone de los servicios esenciales. Hay que conocer las necesidades de la población diana para proveer los recursos adecuados: tratamientos médicos y psiquiátricos, alojamiento, intervenciones rehabilitadoras, actividades de ocio, intervenciones en situación de crisis, medidas de soporte social e incluso asilos.

Diseño individualizado. Cada enfermo mental crónico es único y plantea una constelación particular de necesidades que dependen de sus circunstancias vitales.

Flexibilidad. No es posible la continuidad de cuidados cuando se fuerza al paciente a ajustarse a unos espacios y tiempos estándar. Aunque algunas intervenciones son adecuadas para un paciente en un determinado momento, no quiere decir que exista un único programa o intervención para todos los enfermos mentales siempre. El ritmo y velocidad de movimiento de los pacientes por los elementos de los servicios debe estar determinado por consideraciones clínicas y no por reglas arbitrarias.

Coordinación. Los servicios tienden a estar dispersos entre muchos dispositivos y la responsabilidad muy fragmentada. No es posible la continuidad de cuidados si no existe una coordinación y colaboración real entre los distintos dispositivos y programas que prestan servicio a los pacientes.

Continuidad del terapeuta que permita al paciente establecer una relación estable y de confianza.. Los efectos de la enfermedad mental y las discapacidades generadas aumentan la vulnerabilidad del individuo al aislamiento y a la pérdida de relaciones humanas que le sirvan de apoyo. La continuidad de cuidados no está garantizada si no se cubre este vacío.

Participación del paciente en la planificación del tratamento. No puede existir continuidad de cuidados si no se tienen en consideración las necesidades, deseos, aspiraciones y dudas del sujeto. Estamos abocados al fracaso si se impone un tratamiento con el que el paciente no está de acuerdo.

Pertinencia cultural. Programas que tengan en cuenta la realidad cultural del sujeto. Cada sujeto tiene un conjunto de actitudes, valores y conductas socialmente sancionadas, que influyen en cómo se relaciona con el sistema de atención. Es imposible la continuidad de cuidados si no se comprende y respeta esta orientación cultural.

\section{Modelos de continuidad de cuidados o "case man- agement"}

Bajo el nombre de "case management" o continuidad de cuidados, se engloban distintos programas de intervención a largo plazo con el fin de garantizar la atención de los enfermos mentales graves y crónicos. Aunque se utiliza "case management" para referirse a casi cualquier tipo de programa de apoyo comunitario, a lo largo de los últimos treinta años se han desarrollado distintos modelos entre los que existen grandes diferencias. Como señala Daniel Gélinas, psicoeducador y trabajador social en Quebec (Gélinas, 1998), los programas de continuidad de cuidados se encuentran confrontados desde sus orígenes entre la actividad basada en la coordinación de los servicios y la dimensión clínica de los enfermos mentales, pues sólo la coordinación entre servicios puede convertir el programa en una actividad meramente burocrática. Debate que persiste, durante estas décadas de desarrollo de los programas de continuidad de cuidados en el mundo, reflejando las diferentes concepciones asistenciales y escuelas de los profesionales de la salud mental. Mueser, Bond, Drake y Resnick (1998) revisan y agrupan los modelos de continuidad de cuidados en cinco. Aunque se trata de una simplificación, puesto que existen tantos modelos como equipos los llevan a cabo, permite destacar los elementos diferenciales y más relevantes de cada uno de ellos.

Es el modelo más simple y el primero desarrollado al comienzo de la desinstitucionalización americana en un intento de ayudar a navegar al paciente en el confuso y complejo sistema de salud mental comunitario. El papel del coordinador del caso o "case manager" es valorar las necesidades, desarrollar un plan de cuidados, conectar al paciente con los servicios que pueden prestarle la atención que necesita, supervisar la atención recibida, mantener el contacto y asesorarle (Holloway,1991; Intagliata, 1982). La principal crítica viene de la limitación de los coordinadores ("managers"), a los que este modelo no exige formación clínica, aunque deban afrontar tareas clínicas y supervisar si los proveedores dan los servicios clínicos adecuados.

Este modelo en sentido estricto se ha extendido por todo el mundo, hasta alcanzar la consideración de piedra angular del tratamiento comunitario en los países desarrollados. Los resultados de los estudios comparándolo con un modelo basado en el hospital demostraron desde el principio su superioridad en la reducción de la frecuencia y tiempo de hospitalización, mejora de la calidad de vida y la satisfacción de los pacientes y sus familias (Solomon, 1992). Posteriormente, se ha generado un intenso debate en relación a sus resultados: detractores del modelo han planteado que lejos de reducir la hospitalización la aumentan (Franklin, Solovitz, Mason, Clemons, \& Miller, 1987; Quinlivan et al., 1995; Macias, Kinney, Farley, Jackson, \& Vos, 1994), mientras que Thornicroft, Wykes y sus colaboradores (1998), en los estudios realizados en Reino Unido, consiguen resultados tan exitosos que atribuyen a este modelo efectos 
Tabla 1

Características de los programas de continuidad de cuidados

\begin{tabular}{|c|c|c|c|c|c|}
\hline $\begin{array}{c}\text { Características del } \\
\text { programa }\end{array}$ & Modelo Agencia & Modelo Clínico & $\begin{array}{c}\text { Basado en } \\
\text { Capacidades }\end{array}$ & Modelo Rehabilitador & $\begin{array}{c}\text { Tratamiento } \\
\text { asertivo } \\
\text { comunitario }\end{array}$ \\
\hline Ratio staf: paciente & $1: 50$ & $1: 30+$ & $1: 20-1: 30$ & $1: 20-1: 30$ & $1: 10$ \\
\hline $\begin{array}{l}\text { Seguimiento } \\
\text { pacientes }\end{array}$ & Bajo & Bajo & Moderado & Moderado & Alto \\
\hline Casos compartidos & No & No & No & No & $\mathrm{Si}$ \\
\hline Cobertura 24 horas & No & No & No & No & $\mathrm{Si}$ \\
\hline $\begin{array}{l}\text { Decisión del } \\
\text { paciente }\end{array}$ & No & Baja & Alta & Alta & Baja \\
\hline $\begin{array}{l}\text { Entrenamiento en } \\
\text { habilidades }\end{array}$ & Bajo & Bajo & Moderado & Alto & Moderado \\
\hline $\begin{array}{l}\text { Frecuencia de } \\
\text { contacto con los } \\
\text { pacientes }\end{array}$ & Baja & Moderada & Moderada & Moderada & Alta \\
\hline Lugar de contacto & Consulta & Consulta & Comunidad & Consulta/comunidad & Comunidad \\
\hline $\begin{array}{l}\text { Integración del } \\
\text { tratamiento }\end{array}$ & Baja & Moderada & Baja & Baja & Alta \\
\hline $\begin{array}{l}\text { Provisión directa } \\
\text { de servicios }\end{array}$ & Baja & Moderada & Moderada & Moderada & Alta \\
\hline Población diana & EMG & EMG & EMG & EMG & EMGACR \\
\hline
\end{tabular}

prácticamente similares a los conseguidos por el tratamiento asertivo comunitario a mucho menor coste.

Modelo clínico. Se desarrolla tras el reconocimiento de que el coordinador del caso a menudo debe actuar como clínico prestando directamente el servicio que el paciente necesita. Kanter (1989) plantea cuatro amplias áreas de intervención: Fase inicial (citación, evaluación, planificación); Intervenciones ambientales (conexión con servicios comunitarios, consulta con familiares y otros actores, mantenimiento y ampliación de la red social); Intervenciones con el paciente (psicoterapia individual intermitente, entrenamiento en habilidades de vida independiente, psicoeducación); y Intervenciones con el paciente y su ambiente (intervención en crisis, seguimiento). Este modelo enfatiza la relación entre paciente y tutor como elemento terapéutico esencial y precisa de coordinadores de cuidados con formación clínica en psicoterapia y psicoeducación (Lamb, 1980).

Modelo de fortalezas o capacidades. Se desarrolló en respuesta a la idea generalmente asociada al "case management" y a los tratamientos para personas con enfermedades mentales graves, que sobrevalora los límites o discapacidades secundarios a la enfermedad psiquiátrica y minimiza los recursos individuales. En este modelo se trabaja con las habilidades del individuo en la consecución de metas más que con los déficits. Sus principios los recoge Rapp (1993): Focalizado en las capacidades del individuo más que en lo patológico; La relación coordinadorpaciente es lo esencial y primario; Las intervenciones se basan en la autodeterminación del paciente; La comunidad se ve como un oasis de recursos y no como un obstáculo; El contacto con el paciente ocurre en la comunidad y no en el despacho; Una persona que sufre una enfermedad mental grave puede seguir aprendiendo, creciendo y cambiando.

Modelo rehabilitador. Descrito por Anthony (Anthony, Cohen, Farkas, \& Cohen, 1988), al igual que el anterior, hace hincapié en prestar servicios basados en los deseos y metas individuales de cada paciente, más que en objetivos definidos por el sistema de salud mental. El hecho singular de este modelo es el énfasis en la evaluación y solución de habilidades instrumentales y de vinculación con el entorno, que puedan favorecer la permanencia en la comunidad, y la consecución de los objetivos personales. Distintos trabajos han demostrado la mayor eficacia de este modelo en la mejora del empleo (Bond, 
1992; Stanard, 1999).

Modelo tratamiento asertivo comunitario ("Program for Assertive community treatment", PACT, "Assertive community treatment", ACT o "Assertive Case Management"). Fue desarrollado por Stein y Test (1980) en Madison, Wisconsin, a finales de los años 1960, para responder a las necesidades de los enfermos mentales con psicosis graves y crónicas o con un patrón de elevada frecuentación y uso de recursos, observadas durante el proceso de reforma del Mendota State Hospital. El PACT lo realiza un equipo multidisciplinar, habitualmente compuesto por un psiquiatra, un enfermero y al menos dos coordinadores de caso. Todos participan activamente en la toma de decisiones: la responsabilidad es colectiva. La prioridad en las intervenciones está en prestar soporte práctico a la vida diaria de los enfermos: acompañar al paciente en la toma de medicación, gestionarle beneficios sociales y alojamiento, ir de compras, utilizar transportes, comprar la medicación, ayudarle en la búsqueda de trabajo.

Estos equipos ocupan las tres cuartas partes de su tiempo en las intervenciones directas en el medio natural de los pacientes, reservando el espacio del despacho para las reuniones de coordinación y puesta en común de sus actividades (Brekke $\&$ Test, 1987). Con ello se consigue el entrenamiento en habilidades sociales a partir de situaciones reales en el medio de vida de los pacientes y la programación de actividades ajustadas en intensidad y objetivo a las necesidades cambiantes de los interesados. Así el equipo puede mantener una presencia prolongada al asumir el caso o durante las crisis y reducirla progresivamente. El equipo de Madison, descrito por Test (1998), está formado por 14 profesionales, responsables de la atención de 120 jóvenes adultos diagnosticados de esquizofrenia, disponible las 24 horas del día, los siete días de la semana y con un trabajo localizado principalmente fuera de los despachos. La cobertura de noche (después de las 4 de la tarde) está asegurada por miembros del equipo localizados.

Con más de tres décadas de experiencia, este modelo ha sido muy estudiado, el mejor descrito y más replicado. Desde los estudios iniciales numerosos trabajos han demostrado su eficacia y eficiencia: disminución de la frecuencia y tiempo de hospitalización, mejora en la situación clínica, en la adherencia al tratamiento farmacológico, en la autonomía, en el funcionamiento social, en el empleo protegido, en la calidad de vida, en la relación coste/eficacia y una disminución de la carga familiar (Bond, McGrew, \& Fekete, 1995; Burns \& Santos, 1995; Drake \& Burns, 1995; Latimer, 1999; Marshall \& Lockwood, 2000; McGrew, Bond, Dietzen, McKasson, \& Miller, 1995; Rosenheck, Neale, Leaf, Milstein, \& Frisman, 1995; Test \& Stein, 1980;Wilson, Tien, \& Eaves, 1995). Gracias a estos resultados positivos, el modelo PACT se difundió pronto por EEUU (Deci, Santos, Hiott, Schoenwald, \& Dias, 1995), Canadá (De Cangas, 1994), Australia (Hoult \& Reynolds, 1984) y más tardíamente por Europa (Aberg-Wistedt, Cressell, Lidberg, Liljenberg, \& Osby, 1995; Audini, Marks, Lawrence, Connolly, \& Watts, 1994; Marks et al., 1994) con el mismo tipo de resultados que los obtenidos en el programa original de Madison.

Se han desarrollado normas claras y específicas sobre qué es el PACT e incluso se ha creado un comité internacional de expertos de cara a definir y validar el modelo (McGrew, Bond, Dietzn, \& Salyers, 1994). Se han realizado modificaciones para adaptar el programa a otros contextos como grandes áreas urbanas, en el caso del programa Bridge, del centro de rehabilitación de Thresholds, en Chicago que utiliza el PACT por primera vez en una gran ciudad (Borland et al., 1990; Witheridge \& Dincin, 1985) o el proyecto PRiSM Psychosis Study, que desarrolla y estudia el PACT por un equipo vinculado al Maudsley en dos áreas socioeconómicamente deprimidas del sur de Londres, donde además se compara la eficacia con un modelo estándar de case management (Thornicroft, Wykes et al., 1998) o a zonas rurales (McDonel et al., 1997) o para determinados grupos de riesgo: "homeless" enfermos mentales (Dixon, Krauss, Kernan, Lehman, \& DeForge, 1995; Lehman, Dixon, Kernan, \& DeForge, 1997; Morse et al., 1997; Susser et al., 1997; Wasylenki, Goering, Lemire, Lindsey, \& Lancee, 1993), pacientes con abuso de sustancias (Bond, McDonel, Miller, \& Pensec, 1991; Detrick \& Stiepock, 1992; Drake, Yovetich, Bebout, Harris, \& McHugo, 1997; Durrell, Lechtenberg, Corse, \& Francis, 1993; Lehman, Herron, Schwartz, \& Myers, 1993; Jerrell \& Ridgely, 1995), enfermos en prisión (Challis, Darton, Hughes, Huxley, \& Stewart, 1998; Solomon \& Draine, 1995), niños, pacientes con trastornos de la personalidad.

\section{Objetivos de intervención de los programas de continuidad de cuidados}

\section{Objetivos generales de un programa de apoyo comunitario de acuerdo con Phipps y Liberman (1993):}

Identificar la población diana, tanto en el hospital como en la comunidad, y acceder a ella para brindarle los servicios apropiados. Esto incluye no sólo la población que frecuenta los servicios, si no también la población crónica no detectada.

Estabilización de las crisis en un marco lo menos restrictivo posible.

Proporcionar servicios de rehabilitación psicosocial con establecimiento de objetivos y evaluación de los mismos, alojamiento de transición y rehabilitación laboral.

Facilitar servicios de apoyo de duración ilimitada como alojamiento, oportunidades laborales o actividades diurnas y nocturnas adecuadas a la edad y nivel cultural.

Dar atención médica y psiquiátrica.

Apoyar a las familias, amigos y miembros de la comunidad.

Integrar a personas sensibilizadas en planificación, voluntariado y en la oferta de alojamiento y trabajo.

Proteger los derechos de los pacientes dentro y fuera del hospital y asesorarles para que sean agentes activos en la defensa 
de sus derechos.

Asegurar la disponibilidad del tratamiento apropiado en el recurso adecuado, en cada momento.

\section{Objetivos especificos de los programas de continuidad de cuidados \\ Posibilitar que el paciente pueda manejar sus síntomas.} En este sentido, es de especial importancia el manejo de la medicación, para alcanzar una dosificación adecuada, minimizando los efectos secundarios y enseñando al paciente a sopesar síntomas, efectos secundarios, nivel de funcionamiento y medicación. Se utilizan técnicas de modificación de conducta y cognitivas.

Permitir al paciente cubrir sus necesidades básicas y mejorar su calidad de vida. Teniendo en cuenta las necesidades individuales, mediante experiencias in vivo se estimula al paciente a participar en la vida de la comunidad: buscar un lugar para vivir, lavar, comprar, cocinar, comer en restaurantes, arreglarse, manejar el dinero, usar medios de transporte, encontrar un trabajo, organizar el tiempo libre, desarrollar actividades recreativas y sociales, utilizar servicios sociales.

Apoyar la máxima integración del paciente en la comunidad. La estabilización de los síntomas es el requisito previo para que el paciente defina sus metas (residenciales, laborales, culturales y de funcionamiento social) y adquiera su máxima integración en la comunidad.

Garantizar la integración de los servicios de tratamiento, rehabilitación y soporte social, única forma de asegurar la continuidad de cuidados. Para ello es imprescindible la coordinación entre los distintos recursos y profesionales implicados en el proceso.

Evitar la hospitalización prolongada, acortar la estancia hospitalaria cuando sea imprescindible y prevenir las recaídas. Para ello es crítica la intervención en crisis.

Permitir al paciente adquirir un papel significativo y productivo en la sociedad. Las actividades de rehabilitación profesional son imprescindibles en este sentido. El equipo debe trabajar para que el individuo alcance una ocupación individual estructurada en el mundo real.

Conseguir la confianza del paciente. En este punto es fundamental la orientación cultural del equipo, que debe estar configurado en función del área de referencia asignada.

\section{Éxitos y fracasos de los programas de continuidad de cuidados}

La primera dificultad a la hora de evaluar los éxitos y fracasos está en la diversidad de los programas desarrollados durante estas tres décadas. Aunque en los últimos años se ha hecho un esfuerzo por definir claramente la forma de puesta en marcha y el tipo de intervenciones realizadas, las grandes diferencias originadas por las variaciones en la organización del sistema sanitario y social del Estado, la región o el país, por no hablar de las obvias variaciones en la puesta en marcha de los modelos, hace difícilmente comparables muchos de los resultados. En segundo lugar, los programas referidos en la literatura son en gran parte estudios experimentales realizados en condiciones ideales lo que limita su extrapolación a la práctica habitual y obliga a ser cauto al interpretar los resultados. Por último, debe señalarse que desde el inicio de la desinstitucionalización, las prácticas diarias de intervención en la comunidad se han impregnado y enriquecido con muchos de los elementos aquí descritos, lo que puede reducir la potencia de los hallazgos encontrados al comparar entre los modelos experimental y control (tratamiento comunitario estándar en los centros de salud mental).

Uno de los hallazgos más repetido al aplicar estos modelos es el aumento del número de pacientes que continúan en tratamiento y la reducción de la frecuencia y tiempo de hospitalización. Los primeros programas de case management descritos, los que se limitan a la gestión de casos, a la coordinación entre recursos en manos de aseguradoras (en los que domina el control del gasto), están siendo muy discutidos en la actualidad, ya que los éxitos inicialmente atribuidos, en lo referente a la reducción de los ingresos hospitalarios, reducción de costes y mejora de la evolución de la enfermedad, parecen no diferenciarse de los resultados obtenidos por el tratamiento comunitario estándar.

Actualmente muchos autores los consideran de dudosa eficacia en la mejora clínica, en la reducción de estancias y del gasto, incluso algunos han llegado a señalar un aumento de la hospitalización. Los programas tipo PACT si parecen tener beneficios en aumentar la adherencia al tratamiento, reducir el tiempo de hospitalización, mejorar la convivencia, la estabilidad en la vivienda, la independencia y por ello la calidad de vida, la satisfacción del usuario y de su familia. Menos constantes son los hallazgos en cuanto a la reducción de la gravedad de los síntomas, del abuso de sustancias y del empleo, cuando se comparan con tratamientos estándar (atención ambulatoria en centros de salud mental).

Los programas específicamente orientados a la rehabilitación laboral y el empleo protegido son los únicos que han demostrado incrementar la competitividad laboral de los enfermos mentales graves (McFarlane et al., 1992) los dirigidos al entrenamiento en habilidades sociales son los que más favorecen el ajuste social, mientras que sólo los desarrollados para tratar patología dual parecen ser eficaces en reducir el abuso de sustancias. Los programas tipo PACT mejor dotados, reducen drásticamente el gasto de tratamiento hospitalario, pero cuando se tiene en consideración todo el gasto asociado al tratamiento no se observa una reducción de coste, pero sí un incremento de la eficiencia. Un debate abierto es si el incremento de la eficiencia compensa el incremento de gasto entre los programas estándar y los asertivos comunitarios (Becker, Leese, Clarkson, Szmukler, \& Thornicroft, 1998; Crone et al., 1998; Leese, Johnson, \& Slade, 1998; Marshall et al., 1999; Sashidharan, Smyth, \& Owen, 1999; Szmukler, Wykes, \& Parkman, 1998; Taylor, Leese, Clarkson, Holloway, \& Thornicroft, 1998).

Además de este debate tan vivo, otras líneas de discusión sobre los programas de continuidad de cuidados se centran en: el aumento de horas que los profesionales deben pasar con 
los pacientes con relación a los programas tradicionales; la posibilidad de dar a determinados pacientes un tratamiento más intensivo del que necesitan; la dificultad de interrumpir e incluso reducir la intensidad de la atención; la posibilidad de fomentar la dependencia; la posibilidad de aumentar los problemas legales y de no modificar el tiempo de prisión; la posibilidad de reducir el control del riesgo de suicidio; la falta de delimitación de la población a la que van dirigidos; la contradicción de los datos en relación con la reducción del gasto; dudoso aumento del funcionamiento social (definido por la calidad de las relaciones sociales, el papel que se desempeña y la red social), salvo en programas específicos de entrenamiento en habilidades sociales; y dudosa mejora del desempeño laboral, salvo en programas específicos de formación profesional y/o empleo protegido.

\section{Retos futuros: nuevas líneas de investigación}

Los estudios realizados hasta la fecha se han dirigido a intentar demostrar la superioridad de unos modelos de intervención sobre otros, más que a encontrar los elementos claves de los mismos. A pesar de los avances, seguimos sin conocer los factores últimos responsables del éxito o fracaso de la aplicación de estos modelos, entre otras cosas porque desconocemos la fidelidad de la aplicación de los modelos originalmente descritos en otros lugares. Las investigaciones de atención comunitaria necesitan evaluar esta fidelidad de aplicación para explorar posibles determinantes de la evolución positiva o negativa. Es necesario, por tanto, mejorar los métodos para medir la actividad de los coordinadores de casos y de los equipos multidisciplinares y compararla entre los distintos modelos comunitarios.

Por otra parte, diferenciar entre los distintos modelos de apoyo comunitario y la fidelidad de su aplicación puede servir para identificar los ingredientes críticos. Lo que cada vez parece más claro es que no hay un único modelo de apoyo comunitario que sea igualmente apropiado para cualquier marco de atención (por ejemplo, las características de los recursos rurales frente a los urbanos). Es obvia la necesidad de estudiar el impacto sociodemográfico en el diseño y resultados de los programas comunitarios y sin embargo ha sido escasamente atendida. Otro tema importante de investigación es la relación entre características específicas del paciente y la respuesta a diferentes modelos o facetas de la atención comunitaria (sin techo, patología dual, niños, ancianos). El reto actual sería entender cómo se pueden confeccionar los mejores modelos de intervención para las necesidades específicas de cada paciente.

Los programas de continuidad de cuidados surgieron en respuesta a las necesidades de los enfermos mentales graves, fundamentalmente psicóticos. Aunque posteriormente se han desarrollado programas para poblaciones con otros diagnósticos, sigue siendo un reto el desarrollo de éstos en otras patologías como los trastornos de personalidad o los trastornos de la conducta alimentaria, tremendamente frecuentadores de recursos, pero que encajan mal en el diseño de los desarrollados hasta ahora.

\section{Referencias}

Aberg-Wistedt, A., Cressell, T., Lidberg, Y., Liljenberg, B., \& Osby, U. (1995). Two-year outcome of team-based intensive case management for patients with schizophrenia. Psychiatric Services, 46, 1263-1266.

Anthony, W. A., Cohen, M. R., Farkas, M., \& Cohen, B. F. (1988). Case management - more than a response to a dysfunctional system. Community Mental Health Journal, 24, 219-228.

Audini, B., Marks, I. M., Lawrence, R. E., Connolly, J., \& Watts, V. (1994). Homebased versus out-patient/in-patient care for people with serious mental illness. Phase II of a controlled study. British Journal of Psychiatry, 165, 204-210.

Bachrach, L. L. (1993). Continuity of care and approaches to case management for long-term mentally ill patients. Hospital and Community Psychiatry. 44(5), 465-468.

Becker, T., Leese, M., Clarkson, P., Szmukler, G. I., \& Thornicroft, G. (1998). Impact of community mental health services on users'social networks. PRiSM psychosis study 7. British Journal of Psychiatry, 173, 404-408.

Bond, G. R., McDonel, E. C., Miller, L. D., \& Pensec, M. (1991). Assertive community treatment and reference groups: an evaluation of their effectiveness for young adults with serious mental illness and substance abuse problems. Psychosocial Rehabilitation Journal, 15, 31-43.

Bond, G. R. (1992). Vocational rehabilitation. In R. P. Liberman (Org.), Handbook of psychiatric rehabilitation (pp. 244-275). Nova Iorque: Macmillan Press.

Bond, G. R., McGrew, J. H., \& Fekete, D. M. (1995). Assertive outreach for frequent users of psychiatric hospitals. The Journal of Mental Health Administration, 22, 4-16.

Borland, G. R., Witheridge, T. F., Dincin, J., Wasmer, D., Webb, J., \& De GraafKaser, R. (1990). Assertive Community Treatment for frequent users of psychiatric hospitals in a large city: a controlled study. American Journal of Community Psychology, 18, 865-891.

Brekke, J. S., \& Test, M. A. (1987). An empirical analysis of services delivered in a model community support program. Psychosocial Rehabilitation Journal, 10, 51-61.

Burns, B. J., \& Santos, A. B. (1995). Assertive Community Treatment: An update of randomized trials. Psychiatric Services, 46, 669-675.

Challis, D., Darton, R., Hughes, J., Huxley, P., \& Stewart, K. (1998). Emerging models of care management for older people and those with mental health problems in United Kingdom. Journal of Case Management, 7, 153-160.

Crone, P. M., Thornicroft, G., Phelan, M., Holloway, F., Wykes, T., \& Johnson, S. (1998). Utilization and cost of community mental health services. PRiSM psychosis study 5. British Journal of Psychiatry, 173, 391-398.

De Cangas, J. P. C. (1994). Le “case management" affirmatif: une evaluation complete d'un programme du genre en milieu hospitalier. Sante Mentale au Quebec, 19,75-92.

Deci, P., Santos, A. B., Hiott, D. W., Schoenwald, S., \& Dias, J. K. (1995). Dissemination of assertive community treatment programs. Psychiatric Services, 46, 676-678.

Detrick, A., \& Stiepock, V. (1992). Treating persons with mental illness, substance abuse and legal problems: the Rode Island experience. New Directions in Mental Health Services, 56, 65-77.

Dixon, L. B., Krauss, N., Kernan, E., Lehman, A. F., \& DeForge, B. R. (1995). Modifying the PACT model to serve homeless persons with severe mental illness. Psychiatric Services, 46, 684-688.

Drake, R. E., \& Burns, B. J. (1995). Special section on assertive community treatment: an introduction. Psychiatric Services, 46, 667-668.

Drake, R. E., Yovetich, N. A., Bebout, R. R., Harris, M., \& McHugo, G. J. (1997). Integrated treatmnet for dually diagnosed homeless adults. Journal of Nervous and Mental Disease, 185, 298-305.

Durrell, J., Lechtenberg, B., Corse, S., \& Francis, R. J. (1993). Intensive case management of persons with chronic mental illness who abuse substances. 
Hospital and Community Psychiatry, 44, 415-416.

Franklin, J. L., Solovitz, B., Mason, M., Clemons, J. R., Miller, G. E. (1987). An evaluation of case management. American Journal of Public Health, 77, 674-678.

Gélinas, D. (1998). Points de repère pour différencier la gestion de cas du suivi intensif dans le melieu auprès des personnes souffrant de troubles mentaux graves. Santé mentale au Québec, 13(2), 17-47.

Holloway, F. (1991). Case management for the mentally ill: looking at the evidence. Intenational Journal of Social Psychiatry, 37, 2-13.

Hoult, J., \& Reynolds, I. (1984). Schizophrenia: a comparative trial of community oriented and hospital oriented psychiatric care. Acta Psychiatrica Scandinavia, 69, 359-372.

Intagliata, J. (1982). Improving the quality of community care for the chronically mentally disabled: the role of case management. Schizophrenia Bulletin, 8(4), 655-674.

Jerrell, J., \& Ridgely, M. S. (1995). Comparative effectiveness of three approaches to serving people with severe mental illness and substance abuse disorders. Journal of Nervous and Mental Disease, 183, 566-576.

Kanter, J. (1989). Clinical case management: definition, principles, components. Hospital and Community Psychiatry, 40, 361-368.

Lamb, H. R. (1980). Therapist-case managers: more than brokers of services. Hospital and Community Psychiatry, 31, 762-764.

Latimer, E. A. (1999). Economic impacts of assertive community treatment: a review of the literature. Canadian Journal of Psychiatry, 44, 443-454.

Leese, M., Johnson, S., \& Slade, M. (1998). User perspective on needs and satisfaction with mental health services. PRiSM psychosis study 8 . British Journal of Psychiatry, 173, 409-415.

Lehman, A. F., Herron, J. D., Schwartz, R. P., \& Myers, C. P. (1993). Rehabilitation for adults with severe mental illness and substance use disorders: a clinical trial. Journal of Nervous and Mental Disease, 181, 86-90.

Lehman, A. F., Dixon, L. B., Kernan, E., \& DeForge, B. (1997). A randomized trial of assertive community treatment for homeless persons with severe mental illness. Archives of General Psychiatry, 54, 1038-1043.

Macias, C., Kinney, R., Farley, W. O., Jackson, R., \& Vos, B. (1994). The role of case management within a community support system: partnership with psychosocial rehabilitation. Community Mental Health Journal, 30, 323-339.

Marks, I. M., Connolly, J., Muijen, M., Maudini, G., McNamee, G., \& Lawrence, R. E. (1994). Home-based versus hospital-based care for people with serious mental illness. British Journal of Psychiatry, 165, 179-194.

Marshall, M., Bond, G., Stein, L. I., Shepherd, G., McGrew, J., \& Hoult, J. (1999). PRiSM psychosis study. Design limitations, questionable conclusions. British Journal of Psychiatry, 175, 501-503.

Marshall, M., \& Lockwood, A. (2000). Assertive community treatment for people with severe mental disorders (Cochrane Review). In: The Cochrane Library, Issue 2. Oxford: Update Software.

McDonel, E. C., Bond, G. R., Salyers, M., Fekete, D., Chen, A., McGrew, J. H., \& Miller, L. (1997). Implementing assertive community treatment in rural settings. Administration and Policy in Mental Health, 25, 153.173.

McFarlane, W. R., Stastny, P., \& Deakins, S. (1992) Family-aided assertive community treatment: a comprehensive rehabilitation and intensive case management approach for persons with schizophrenic disorders. New Directions for Mental Health Services, 53, 43-54.

McGrew, J. H. , Bond, G. R., Dietzn, L., \& Salyers, M. (1994). Measuring the fidelity of implementation of a mental health program model. Journal of Consulting and Clinical Psychology, 62, 670-678.

McGrew, J. H., Bond, G. R., Dietzen, L., McKasson, M., \& Miller, L.D. (1995). A multisite study of client outcomes in assertive community treatment. Psychiatric Services, 46, 696-701.

Morse, G. A., Calsyn, R. J., Klinkenberg, W. D., Trusty, M. L., Gerber, F., Smith, R., ... Ahmad, L. (1997). An experimental comparison of three types of case management for homeless mentally ill persons. Psychiatric Services, 48, 497-503.
Mueser, K. T., Bond, G. R., Drake, R. E., \& Resnick, S. G. (1998). Models of community care for severe mental illness: a review of research on case management. Schizophrenia Bulletin, 24(1), 37-74.

National Institute of Mental Health. Division of Biometry and Epidemiology (1977). Data sheet on State and County Mental Hospitals. Rockville: NIMH.

Phipps, C., \& Liberman, R. P. (1993). Apoyo comunitario. In R. P. Liberman (Org.), Rehabilitación integral del enfermo mental crónico (pp.230-249). Barcelona: Martínez Roca.

Quinlivan, R., Hough, R., Crowell, A., Beach, C., Hofstetter, R., \& Kenwothy, K. (1995). Service utilization and cost of care for severely mentally ill clients in an intensive case management program. Psychiatric Services, 46, 365-371.

Rapp, C. A. (1993). Theory, principles and methods of the strengths model of case management. In M. Harris \& H. C. Bergman (Orgs.), Case management for mentally ill patients: theory and practice (pp. 143-164). Langhorne: Harwood Academic Publishers.

Rosenheck, R., Neale, M., Leaf, P., Milstein, R., \& Frisman, L. (1995) Multisite experimental cost study of intensive psychiatric community care. Schizophrenia Bulletin, 21, 129-140.

Sashidharan, S. P., Smyth, M., \& Owen, A. (1999). PRiSM psychosis study. Thro'aplass darkly: a distorted appraisal of community care. British Journal of Psychiatry, 175, 504-507.

Solomon, P. (1992). The efficacy of case management services for severely mentally disabled clients. Community Mental Health Journal, 28, 163-180.

Solomon, P., \& Draine, J. (1995). One-year outcomes of a randomized trial of case management with seriously mentally ill clients leaving jail. Evaluation Review, 19, 256-273.

Stanard, R. P. (1999). The effect of training in a strengths model of case management on clients outcomes in a community mental health center. Community Mental Health Journal, 35, 169-179.

Stein, L. I., \& Test, M. A. (1980). Alternative to mental hospital treatment: conceptual model, treatment program and clinical evaluation. Archives of General Psychiatry, 37, 392-397.

Susser, E., Valencia, E., Conover, S., Felix, A., Tsai, W. Y., \& Wyatt, R. J. (1997) Science and homelessness: critical time intervention for mentally ill men. American Journal of Public Health, 87, 256-262.

Szmukler, G. I., Wykes, T., \& Parkman, S. (1998). Care-giving and the impact on carers of a community mental health services. PRiSM psychosis study 6. British Journal of Psychiatry, 173, 399-403.

Taylor, R., Leese, M., Clarkson, P., Holloway, F., \& Thornicroft, G. (1998) Quality of life outcomes for intensive versus standard community mental health services. PRiSM psychosis study 9. British Journal of Psychiatry, 173, 416-422.

Test, M. A., \& Stein, L. I. (1980). Alternative to mental hospital treatment. III Social cost. Archives of General Psychiatry, 37,409-412.

Test, M. A. (1998). Modèles de traitement dans la communauté pour adultes ayan des maladies mentales graves et persistantes. Santé mentale au Québec, 23, 119-143.

Thornicroft,G., Strathdee, G., \& Phelan, M. (1998). Rationale and design. PRiSM psychosis study I. British Journal of Psychiatry, 173, 363-370.

Thornicroft, G., Wykes, T., Holloway, F., Jonhson, S., \& Szmukler, G. (1998). From efficacy to effectiviness in community mental health services. PriSM Psychosis Study 10. British Journal of Psychiatry, 173, 423-427.

Turner, J. C., \& Ten Hoor, W. J. (1978). The NIMH Community Support Program: Pilot approach to social reform. Schizophrenia Bulletin, 4(3), 319-348.

Wasylenki, D. A., Goering, P.N., Lemire, D., Lindsey, S., \& Lancee, W. (1993). The hostel outreach program: assertive case management for homeless mentally ill persons. Hospital and Community Psychiatry, 44, 848-853.

Wilson, D., Tien, G., \& Eaves, D. (1995). Increasing the community tenure of mentally disordered offenders: an assertive case management program. International Journal of Law and Psychiatry, 18, 61-69.

Witheridge, T. F., \& Dincin, J. (1985). The Bridge: an assertive outreach program in an urban setting. New Directions for Mental Health Services, 26, 65-76. 
312 A. G. Rodríguez

Ana González Rodríguez é psiquiatra, responsável pelo programa de continuidade de cuidados do Centro de Salud Mental de Chamartín, Madrid. Endereço para correspondencia: C/ Juan de Austria 30; 4 A. 2010, Madrid. Telefone: (34) 914486102. E-mail: ana.gonzr@gmail.com 